\title{
A revised distance scale of planetary nebulae ${ }^{\star}$ (Research Note)
}

\author{
Roberto Ortiz \\ Escola de Artes, Ciências e Humanidades, USP, Av. Arlindo Bettio 1000, 03828-000 São Paulo SP, Brazil \\ e-mail: rortiz@usp.br
}

Received 20 June 2013 / Accepted 25 October 2013

\begin{abstract}
Context. The advent of large-scale, high-sensitivity surveys of the Galaxy (2MASS, WISE, Spitzer, etc.) has allowed detection of a large number of planetary nebulae (PNe), some of them at large distances.

Aims. I propose a new distance scale of planetary nebulae, based on two main quantities: the flux density (or magnitude) at $8 \mu \mathrm{m}$ and the nebular apparent size.

Methods. I used these quantities to calculate the specific intensity of the radiation $I_{8 \mu \mathrm{m}}$, which decreases as the nebula evolves. The PNe situated in the Large Magellanic Cloud (LMC) with $8 \mu \mathrm{m}$ photometry obtained by Spitzer and diameter obtained from HST observations were used as calibrators.

Results. After calibration, I recalculated distances of PNe in the LMC, and they show a one-sigma spread of $10 \mathrm{kpc}(20 \%)$. Eventually, I applied the method to a sample of 47 Galactic PNe observed during the Spitzer survey. These results showed agreement within 0.2 dex with the distances determined with another method of calibrating with PNe in the LMC.

Conclusions. The main advantage of the present distance scale is that it is nearly "extinction free", which makes it especially suited to PNe severely affected by interstellar extinction, such as the Galactic disc and bulge. On the other hand, the method requires an unbiased determination of the nebular size, which might be quite uncertain for PNe with extended faint features, such as haloes and ansae.
\end{abstract}

Key words. planetary nebulae: general - infrared: ISM - stars: AGB and post-AGB - stars: distances - Galaxy: structure

\section{Introduction}

Since the pioneering work by Shklovsky (1956), different attempts to determine the distances of PNe have been proposed with a wide variety of techniques. These methods can be divided into two main classes: (1) the statistical methods make use of the relationships among general characteristics of PNe (quantities that are distance-invariant are used to infer other physical quantities that depend on the distance, such as constant ionized mass, Shklovsky 1956; the radio flux, Milne \& Aller 1975; the brightness temperature - nebular radius correlation, Zhang \& Kwok 1993); and (2) individual methods, which apply to specific physical characteristics relative to the nebula or its central star. At first glance, individual methods seem to be more reliable. However, even among these direct methods, large discrepancies still exist. Thus, when correctly proposed, statistical methods still constitute a valid approach to the problem.

In an earlier paper, Ortiz et al. (2011) proposed a distance scale based on a minimal set of infrared data: the mid-infrared specific intensity $I_{\lambda}$ and the apparent size, $\theta$. Although the present method is based on the same set of physical quantities as used in Ortiz et al. (2011), the dependence among these parameters and the distance of $\mathrm{PNe}$ is modelled differently.

The discrepancy between some of the distances determined by Stanghellini et al. (2008, hereafter SSV) and Ortiz et al. (2011) also demanded a revision of the method. Some bias seems to affect the nearer objects, especially those within $2 \mathrm{kpc}$. For

* Tables $1-3$ are available in electronic form at http://www . aanda.org these PNe, the distances determined by Ortiz et al. (2011) were clearly overestimated by a factor 2 , on average. Besides this, another bias seems to affect the farther objects: PNe with $d>$ $7.5 \mathrm{kpc}$ had their distances underestimated by $30-50 \%$ by Ortiz et al. (2011). Thus, a revision of the method became necessary, and this is the main goal of the present paper.

\section{The method and its calibration}

Like many of the distance scales previously described in the literature, the present method is based on a physical quantity that remains invariant with the distance: the specific intensity of the radiation, $I_{\lambda}$. The first studies of the behaviour of $I_{\lambda}$ of PNe are from Volk (1992) and Zhang \& Kwok (1993) who showed that $I_{\lambda}$ of the IRAS bands keep a tight correlation with the brightness temperature measured at radio wavelengths. Van de Steene \& Zijlstra (1994) developed a statistical distance scale based on an empirical correlation between the radio-continuum brightness temperature and the nebular radius. Eventually, Ortiz et al. (2011) derived a similar correlation for several wavelengths between 8 and $21 \mu \mathrm{m}$. According to these studies, as a nebula evolves, its optical depth at radio wavelengths decreases as the result of the geometrical dilution of the gas. At the same time, the specific intensity at mid-infrared wavelengths also decreases, approximately at the same rate.

Another physical parameter that varies monotonically with time is the nebular radius. Our present knowledge about this matter includes the interaction (or shock) of different stellar winds (Kwok et al. 1978) - each of them with its own distinct 
velocity - with the local interstellar medium (Wareing et al. 2007). All the studies of this matter point out that the central star wind must have at least two components: a slow wind, which is remmant of the asymptotic giant branch (AGB) phase, showing $V_{\exp }=10-20 \mathrm{~km} \mathrm{~s}^{-1}$ (Mufson et al. 1975; Kwok 1982); a fast wind, revealed by the broad P-Cygni profiles of resonance lines observed in the UV, showing velocities as high as 2000-4000 $\mathrm{km} \mathrm{s}^{-1}$ (Heap et al. 1978). Besides these two components, PNe are also shaped by additional physical mechanisms, which generate the complex structures, such as jets, knots, and tori.

As formerly defined in Ortiz et al. (2011), the specific intensity at any wavelength can be evaluated according to the following equation:

$I_{\lambda}=5.416 \times 10^{4} \frac{F_{\lambda}}{\theta_{\lambda}^{2}}$,

where $I_{\lambda}$ is given in MJy sr${ }^{-1}, F_{\lambda}$ is the flux density in Jansky, and $\theta_{\lambda}$ the apparent nebular diameter, in arcseconds. The infrared flux density in the equation above can be converted into magnitude units using the IRAC zero-magnitude flux density at $8 \mu \mathrm{m}$, $F_{\text {zero, } 8 \mu \mathrm{m}}=64.13$ Jansky (Meade et al. 2009). After conversion, Eq. (1) can be rewritten as

$\log I_{8 \mu \mathrm{m}}=6.541-0.4 m_{8 \mu \mathrm{m}}-2 \log \theta_{8} \mu \mathrm{m}$,

where $m_{8} \mu \mathrm{m}$ refers to the magnitude corresponding to $F_{8} \mu \mathrm{m}$.

The distance scale proposed in this paper is calibrated with a sample of PNe situated in the Large Magellanic Cloud (LMC). All the $8 \mu \mathrm{m}$ magnitudes of the calibrators were extracted from Hora et al. (2008). They were calculated from the solid angleintegrated intensity of IRAC images of previously identified PNe in the LMC-SAGE survey (Meixner et al. 2006).

\subsection{The relationship between visual and $8 \mu \mathrm{m}$ apparent sizes}

As formerly discussed in Ortiz et al. (2011), the main source of uncertainty for any distance scale based on the parameters above is the definition of the nebular apparent size. Because PNe are diffuse and often display faint haloes around a prominent nebular bulk (Jewitt et al. 1986), the evaluation of $\theta_{\lambda}$ depends on various factors, such as the wavelength of the observation, the sensitivity and spatial resolution of the camera, and the morphology of the nebula. The compilation of a sample consisting of PNe situated only in the LMC imposes additional difficulties, especially concerning the sensitivity and spatial resolution of the observations. Shaw et al. (2007) discussed the discrepancy between LMC nebular sizes obtained from HST images and with ground-based telescopes. According to that study, faint, outer haloes may be overlooked in HST images. Besides, PNe with angular diameter smaller than the spatial resolution of ground-based telescopes have their sizes overestimated.

Unfortunately, the spatial resolution of the IRAC camera at $8 \mu \mathrm{m}$ does not allow correct determination of $\theta_{8} \mu \mathrm{m}$ of LMC$\mathrm{PNe}$, and other determinations of this quantity still do not exist in the literature. It is well known that nebular apparent sizes depend on the wavelength, but these differences may not be significant in all cases. Bedding \& Zijlstra (1994) compared nebular diameters obtained from images taken with the $\mathrm{H} \alpha$ filter with those determined from $6 \mathrm{~cm}$ radio observations with the VLA. They concluded that, even though the agreement between these quantities is very poor when only compact $\left(<10^{\prime \prime}\right)$ objects are selected, $\theta_{\mathrm{rad}}$ generally equals $\theta_{\text {vis }}$ for larger $\left(>10^{\prime \prime}\right)$ objects. Ortiz et al. (2011)

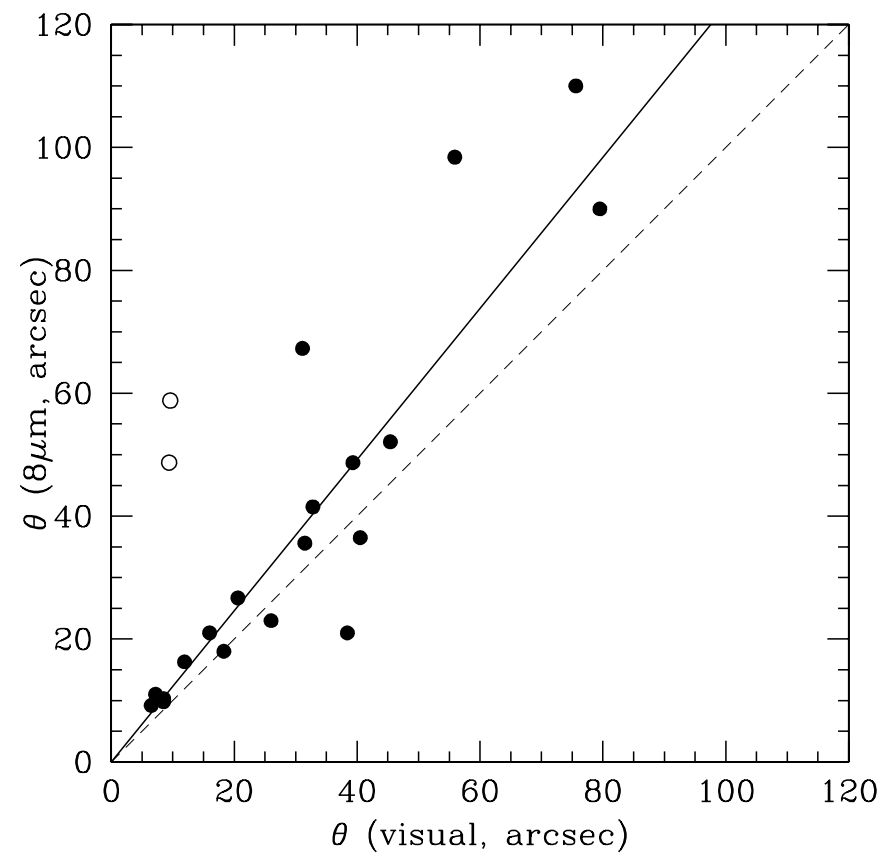

Fig. 1. Filled circles represent apparent sizes of Galactic PNe, measured at both $8 \mu \mathrm{m}$ and visual wavelengths. The continuous straight line represents the best fit to the data, excluding two objects with $\theta_{8 \mu \mathrm{m}} / \theta_{\mathrm{vis}}>3$ (plotted as open circles).

also compared sizes obtained at radio (the VLA, Siódmiak \& Tylenda, 2001) with diameters measured at visual wavelengths (the compilation by SSV). Their results showed good agreement in $85 \%$ of the cases, confirming the previous results by Bedding \& Zijlstra (1994). A comparison among sizes determined at different wavelengths in the visual range was carried out by Ruffle et al. (2004). They compared diameters obtained from images taken both at $\mathrm{H} \alpha$ and $\mathrm{O}[\mathrm{III}]$ wavelengths and showed that these quantities agree within $15 \%$ in more than $90 \%$ of the cases.

This scenario changes completely when nebular sizes obtained at visual and mid-infrared wavelengths are compared. Some nebulae show dusty envelopes far beyond the ionization front, a relic of the intense mass loss that occurred at the end of the AGB. Because this outer envelope contains large amounts of warm dust, its emission peaks at mid-infrared wavelengths. Thus, the nebular size used in the calculation of $I_{8} \mu \mathrm{m}$ must be measured or estimated at this same wavelength. In this work the nebular size of LMC-PNe at $8 \mu \mathrm{m}$ is estimated from its visual value. I analysed a sample of Galactic PNe observed at $8 \mu \mathrm{m}$ in order to obtain a relationship between $\theta_{\text {vis }}$ and $\theta_{8} \mu \mathrm{m}$ (Table 1).

Figure 1 shows a comparison between apparent sizes of Galactic PNe observed by Spitzer and the sizes obtained at visual wavelengths ${ }^{1}$. The $\theta_{8} \mu \mathrm{m}$ values were obtained from $\theta_{8} \mu \mathrm{m}, 10 \%$, which was taken from isophotal maps and/or profile cuts, and deconvolved according to the following relationship proposed by Tylenda et al. (2003):

$\theta_{8 \mu \mathrm{m}}=\sqrt{\theta_{8 \mu \mathrm{m}, 10 \%}^{2}-\left(1.823 \phi_{b}\right)^{2}}$

where $\phi_{b}$ is the full width at half maximum (FWHM) of the resolution beam. Kwok et al. (2008) estimated the FWHM of the point-spread function of the IRAC camera at $8 \mu \mathrm{m}$ as $\phi_{b}=$ $1.94^{\prime \prime}$. A least-squared fit to the data shown in Fig. 1 resulted

1 In the case of non-spherical shape, $\theta$ is calculated as $\theta=\sqrt{a \times b}$, where $a$ and $b$ represent the major and minor nebular dimensions. 
in $\left\langle\theta_{8 \mu \mathrm{m}} / \theta_{\mathrm{vis}}\right\rangle=1.23 \pm 0.34$. Two ( $10 \%$ of the sample) compact $\left(<10^{\prime \prime}\right)$ PNe were not included in the calculation because of their discrepant diameters $\left(\theta_{8} \mu \mathrm{m} / \theta_{\text {vis }}>3\right)$.

Sizes of LMC-PNe were determined by Shaw et al. (2001, 2006) from various HST images taken at broadband, $\mathrm{O}[\mathrm{III}] \lambda 5007$, and $\mathrm{H} \alpha+\mathrm{N}[\mathrm{II}]$ images. The apparent diameter of a round nebula is defined as the isophotal diameter taken at the level of $10 \%$ of the maximum intensity. In the cases where the nebular shape deviates from a round shape, the diameter was calculated as the square root of the product of the larger and smaller dimensions (the major and minor axes, assuming the PN to be elliptical). After deconvolution, the spatial resolution of HST images is only $\sim 0.1^{\prime \prime}(\sim 0.02$ parsec at $50 \mathrm{kpc}$ of distance, Mould et al. 2000), which allows including of a wide range of nebular sizes.

Unfortunately, high-resolution images of LMC-PNe still do not exist at mid-infrared wavelengths, and consequently their diameters at $8 \mu \mathrm{m}$ can only be estimated from the values obtained in visual bands. In this study, I used the relationship between visual and $8 \mu \mathrm{m}$ apparent diameters of Galactic PNe (Fig. 1) to estimate the $8 \mu \mathrm{m}$ diameters of LMC-PNe. Because these data show a considerable spread, $8 \mu \mathrm{m}$ diameters cannot be calculated by simply multiplying the visual sizes by the $\left\langle\theta_{8} \mu \mathrm{m} / \theta_{\mathrm{vis}}\right\rangle$ average ratio. Instead, artificial $8 \mu \mathrm{m}$ diameters were generated by a Monte Carlo simulation applied to the visual diameters of LMCPNe given by Shaw et al. (2001, 2006), assuming that these data follow a Gaussian distribution with $\left\langle\theta_{8} \mu \mathrm{m} / \theta_{\mathrm{vis}}\right\rangle=1.23 \pm 0.34$.

Table 2 shows various characteristics of $\mathrm{PNe}$, as well as the results of the Monte Carlo method used in the calibration. Nebular sizes at $8 \mu \mathrm{m}\left(\theta_{8} \mu \mathrm{m}\right)$ are not individually valid because they represent sizes calculated by the Monte Carlo simulation. Therefore, $8 \mu \mathrm{m}$ sizes are artificial, and have been included to simulate the distribution of mid-infrared sizes of LMC-PNe in the diagram, which do not exist in the literature.

Figure 2 shows the results of this technique. For each nebula, a second $I_{8} \mu \mathrm{m}$ was calculated by substituting $\theta_{\text {vis }}$ by $\theta_{8} \mu \mathrm{m}$, generated by a Monte Carlo simulation. These $8 \mu \mathrm{m}$ data are plotted in Fig. 2 according to the morphological type of the nebula.

An analysis of Fig. 2 reveals that (1) the shift caused by exchanging $\theta_{\text {vis }}$ by $\theta_{8} \mu \mathrm{m}$ in the calculation of $I_{8} \mu \mathrm{m}$ is, on average, almost parallel to the distribution of data, i.e. the diagonal in the diagram (2) Worst-fit data correspond mostly to bipolar nebulae, confirming the previous conclusion by SSV, who state that distance scales of this kind do not work well for bipolar PNe; (3) there is only one PN (SMP11, near $\log D(\mathrm{pc}) \simeq 0.75$ and $\left.\log I_{8 \mu \mathrm{m}}\left(\mathrm{MJy} \mathrm{sr}^{-1}\right) \simeq 4.5\right)$ in disagreement with the fit curve. This object is described by Shaw et al. (2006) as one of the smallest known bipolar PNe in the LMC. A misevaluation of its diameter might have been the cause of its detached position in the diagram. (4) All PNe situated near the bottom right-hand corner of the diagram have been classified as bipolar or other than round or elliptical. The combination of large diameter with low-intensity radiation (near the threshold detecting level of the HST images) may have caused the overestimation of $I_{8} \mu \mathrm{m}$.

\subsection{Estimation of the physical nebular diameter from the intensity of the nebular radiation at $8 \mu \mathrm{m}$}

The plot shown in Fig. 2 reinforces the interpretation formerly proposed by Zhang \& Kwok (1993) which states that $I_{\lambda}$ at midinfrared wavelengths decreases as the nebula evolves. The data situated near the top left-hand corner of Fig. 2 correspond to young, compact PNe. The most compact objects in the present sample show $\log I_{8 \mu \mathrm{m}} \simeq 4.7$, similar to $\log I_{8 \mu \mathrm{m}} \simeq 4.8$ observed

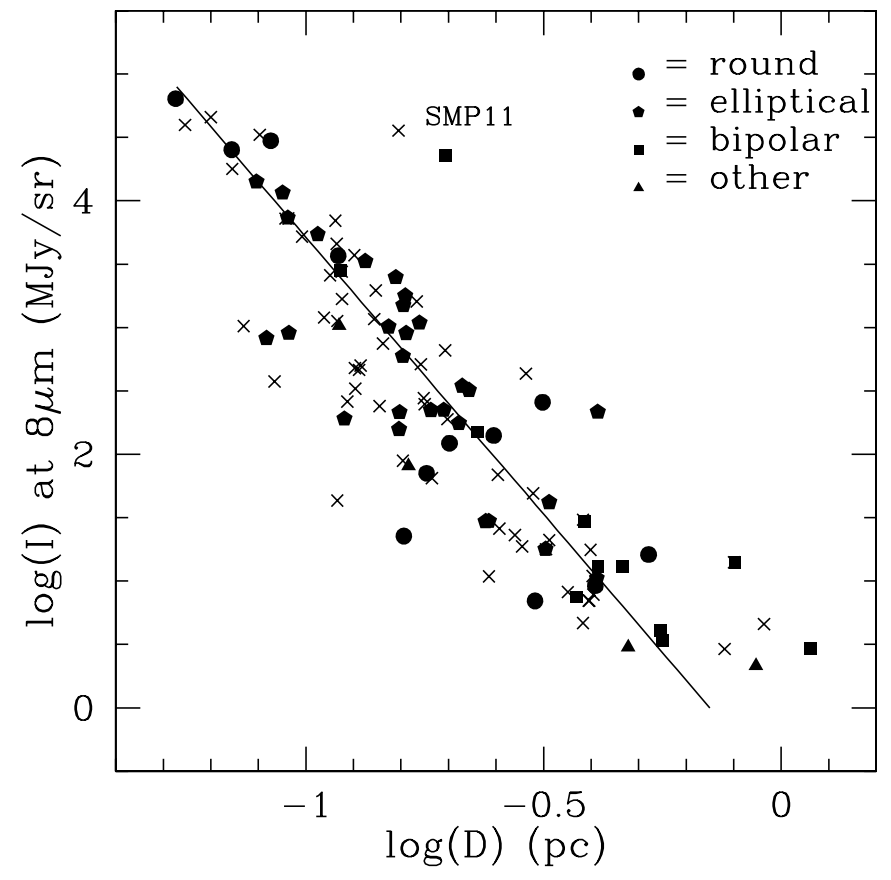

Fig. 2. Statistically adjusted nebular diameter ( $D$, in parsecs) versus the specific intensity of the radiation at $8 \mu \mathrm{m}$. Crosses represent the intensity at $8 \mu \mathrm{m}$, calculated with the visual nebular size $\theta_{\text {vis }}$. Each filled symbol was generated by a Monte Carlo simulation that transformed the original data $\theta_{\text {vis }}$ into $\theta_{8 \mu \mathrm{m}}$, assuming $\theta_{8 \mu \mathrm{m}} / \theta_{\mathrm{vis}}=1.23 \pm 0.34$.

in the sample compiled by Ortiz et al. (2011), carried out at a similar wavelength. At the other end of the sequence, where there are PNe showing low $I_{8} \mu \mathrm{m}$, a comparison between the present data with the sample studied by Ortiz et al. (2011) confirms the higher sensitivity of the Spitzer IRAC camera compared to the Spirit instrument used in the MSX survey.

The following equation has been obtained from a leastsquared fit to the data shown in Fig. 2:

$\log \hat{D}(\mathrm{pc})=-0.150( \pm 0.039)-0.229( \pm 0.015) \log I_{8} \mu \mathrm{m}$

where $\hat{D}$ represents the estimated diameter of the nebula, obtained from its $I_{8} \mu \mathrm{m}$. The correlation coefficient of the fit is $R^{2}=$ 0.804. One nebula, SMP11, was excluded from the fit owing to its divergent position in the diagram.

Equation (4) is similar to the relationship between the nebular radius and the brightness temperature in the radio domain, which is used to calibrate other distance scales (van de Steene \& Zijlstra 1995, SSV). However, as pointed out by Urosevic et al. (2009), there are large discrepancies among the various distance scales based on relationships of this kind. According to their analysis, the slope of brightness-radius relationship varies according to the sample used in the calibration. Slopes vary from -0.25 (for PNe with $d>2 \mathrm{kpc}$, SSV) up to -0.68 (for nearby PNe, with $d<0.4 \mathrm{kpc}$, SSV and Phillips 2002). The slope in Eq. (4) is distinct from the values determined with other distance scales, but this may result from the different hypotheses assumed by the other methods, such as the definition of angular size.

Once the nebular diameter is estimated, the distance $d$ of a PN can be estimated from the well-known relationship:

$d(\mathrm{kpc})=206.265 \frac{\hat{D}(\mathrm{pc})}{\theta\left({ }^{\prime \prime}\right)}$ 
The substitution of the previous equations into the relationship above results in

$d=4.639 \theta_{8 \mu \mathrm{m}}^{-0.542} 10^{+0.0916\left(m_{8} \mu \mathrm{m}-0.024 A_{V}\right)}$,

where $d$ is the distance (in kiloparsecs), $\theta_{8} \mu \mathrm{m}\left({ }^{\prime \prime}\right)$ the apparent size (diameter, or its equivalent) at $8 \mu \mathrm{m}, m_{8} \mu \mathrm{m}$ is the nebular magnitude at $8 \mu \mathrm{m}$ (Spitzer), and $A_{V}$ the visual extinction of the nebula.

\subsection{Estimation of errors}

This method contains intrinsic shortcomings that produce errors in the distance. Figure 2 shows the data distributed along the fitted curve with a one-sigma spread of $\sim 0.2 \mathrm{dex}$ in $\log (D)$. This scattering is the main source of error in the present method and is caused mainly by (1) errors in the $\theta$ measurements of the LMC-PNe, i.e. the calibration; (2) intrinsic variation in $I_{8} \mu \mathrm{m}$, due to the various features seen among PNe spectra. These variations include the intensity of the continuum (which depends on thermal emission by dust), the strength of emission molecular bands caused by various chemical compounds in the nebula, especially those deposited onto grains, etc. The uncertainty intrinsic to the method (the 0.2 dex scattering in Fig. 2) directly affect the distance calculated by Eq. (5), resulting in a one-sigma error bar of $d_{-37 \%}^{+58 \%}$.

A second cause of error in the distance is the misevaluation of the nebular angular diameter, but this does not affect all $\mathrm{PNe}$ equally. Figure 2 shows that bipolar or peculiar-type PNe are more subject to this error. Misevaluation of nebular sizes can also result from observational factors, such as instrumental limitations and low signal-to-noise ratio of the image. As an example, if the angular diameter was overmeasured by $20 \%$, its estimated diameter $(\hat{D})$ would be $8.7 \%$ larger than its real size (by Fig. 2 and Eq. (4)). However, its distance, calculated according to Eq. (6), would be underestimated by $10 \%$. On the other hand, a misevaluation of the angular diameter by $20 \%$ less than its real size would produce a distance in excess of $13 \%$.

The interstellar extinction is another factor that can affect determination of the distance, especially those PNe located in the Galactic bulge. Schultheis et al. (1999) estimate the average visual extinction up to the Galactic centre as $25^{\mathrm{m}}$. When assuming $A_{8 \mu \mathrm{m}}=0.024 \times A_{V}(\mathrm{Li} \&$ Greenberg 1997; Cox 2000), the extinction at $8 \mu \mathrm{m}$ to the Galactic bulge would amount to $0.6^{\mathrm{m}}$, which would cause the distance to be overestimated by $13 \%$. In this study, only Pe 2-12 might have had its distance affected a little by interstellar extinction. Generally, this effect has little influence on the determination of disc PNe.

There are only a few distance determinations obtained with "direct" methods that could be used as a independent calibration, such as trigonometric and expansion parallaxes and binary planetary nebula nucleii. Statistical methods rely on physical hypotheses with various restrictions: PNNi spectroscopic distances (Méndez et al. 1988), constant ionized mass (Shklovsky 1956), etc. Unfortunately a comparison between the "direct" and the statistical methods is very difficult to make, especially because there are only a few objects with distances determined by both types of methods. For example, until now there have only been four PNe near the Galactic plane $\left(|b|<5^{\circ}\right)$ with distances determined by trigonometric parallax, NGC 6853 (Benedict et al. 2009; Harris et al. 2007), NGC 6881 (Gúzman-Ramírez et al. 2011), Sh 2-216 (Harris et al. 2007), and Hen 2-11 (Gutierrez-Moreno et al. 1999), but none of these objects was observed in the GLIMPSE survey. There are also a few objects

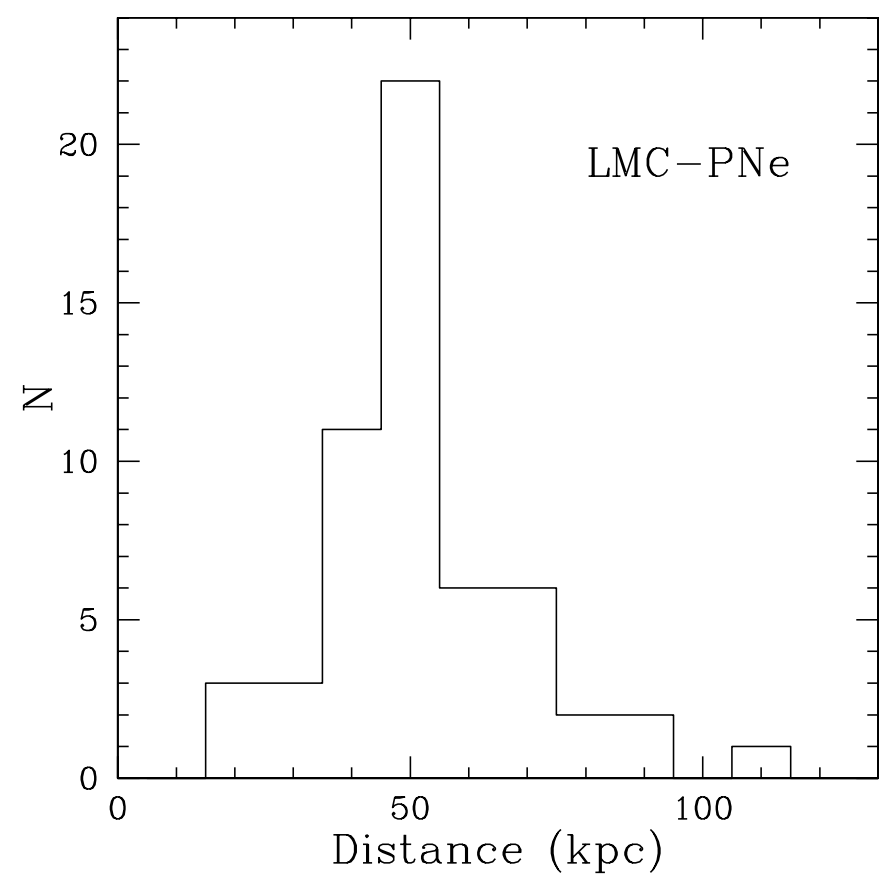

Fig. 3. Histogram, showing the distance of PNe situated in the LMC, determined by the present method. The sample consists of the same set as used to compose the data shown in Fig. 2.

with distances obtained by expansion parallaxes observed by GLIMPSE. However this method also has its limitations, especially concerning the derivation of the nebular expansion velocity (Mellema 2004), and the distances determined by this method may be underestimated by a factor $1.3 \sim 3$ (Schoenberner et al. 2005).

\section{Results and discussion}

To test the new distance scale, I applied the method to PNe situated in the LMC and the Galaxy. The first test was to recalculate the distance of LMC-PNe using the same data set as was used to compose the calibration obtained in Sect. 2. The histogram depicted in Fig. 3 peaks at $d=50 \mathrm{kpc}$ and has a half width of $\sim 10 \mathrm{kpc}$. This scatter, corresponding to a relative uncertainty of $20 \%$, is mainly due to the dispersion of the data near the fitted curve shown in Fig. 2, as discussed in Sect. 2.3. The distance of a few objects have been severely under- or overestimated in this test. SMP11, the discrepant data point in Fig. 2 has had its distance underestimated as $18.1 \mathrm{kpc}$. On the other hand, four objects have clearly overestimated distances: SMP80, SMP82, SMP84, and MG40 (with distances of 107.9, 92.0, 80.8, and $88.3 \mathrm{kpc}$, respectively). Among them, only MG40 seems to exhibit special morphological features: a bipolar core and an attached outer shell (Shaw et al. 2006). SMP84 has a visual diameter of $0.57 \times 0.48^{\prime \prime}$ (Shaw et al. 2006), but its distance was calculated assuming a $8 \mu \mathrm{m}$ diameter of $0.380^{\prime \prime}$ generated by the Monte Carlo simulation. This is one of the very few cases where the $8 \mu \mathrm{m}$ size is smaller than its visual value. If the average $\left\langle\theta_{8} \mu \mathrm{m} / \theta_{\text {vis }}\right\rangle=1.23$ ratio had been used to calculate $\theta_{8} \mu \mathrm{m}$ instead of generating a $8 \mu \mathrm{m}$ size by the Monte Carlo simulation, its distance would be calculated as $60.7 \mathrm{kpc}$. SMP82, showing $\theta_{\text {vis }}=0.305^{\prime \prime}$ is among the four smallest objects in the sample, resulting in the large relative uncertainty of its size. SMP-80 is the faintest PN in the sample, resulting in the large uncertainty of its $8 \mu \mathrm{m}$ magnitude and consequently $I_{8} \mu \mathrm{m}$.

A second test of the method consisted in determining the distances of Galactic PNe. Different authors have measured the 

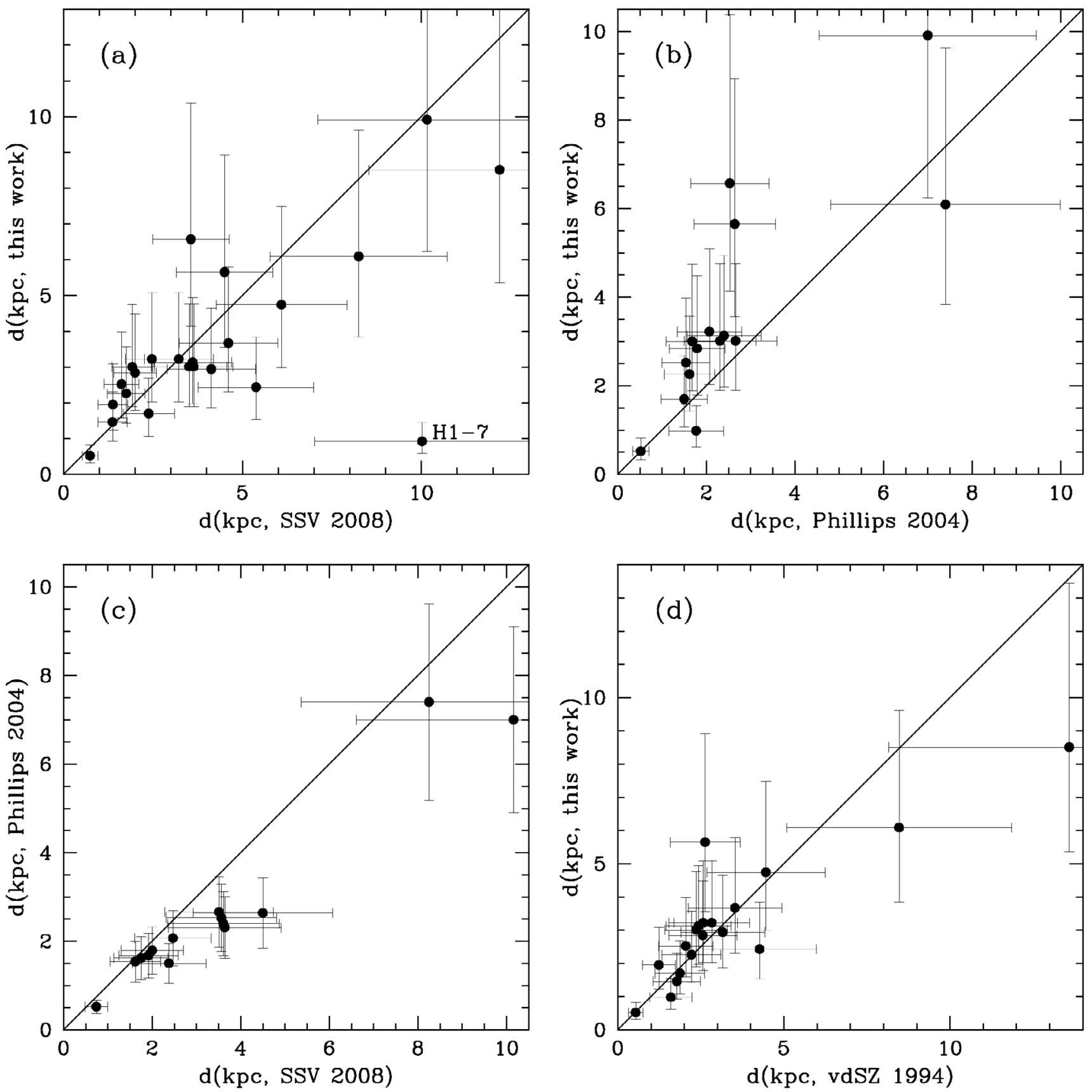

Fig. 4. Comparison between the distances obtained in the present work with others: a) Stanghellini et al. (2008, SSV); b) Phillips (2004); c) SSV versus Phillips (2004); d) van de Steene \& Zijlstra (1994). The error bars correspond to the average value in the original reference, whilst the error bar of this method corresponds to its minimum value: 0.2 dex or $d_{-37 \%}^{+58 \%}$.

total flux density and size of these nebulae, and a compilation of data collected from the literature resulted in $47 \mathrm{PNe}$ with $8 \mu \mathrm{m}$ magnitudes and diameters (Table 3 ). In the cases where the $8 \mu \mathrm{m}$ intensity was given by brightness profiles or isophote maps, the outer border was defined at the level corresponding to one tenth of the maximum intensity.

The validity of a distance scale calibrated with LMC-PNe but applied to Galactic objects can be questioned if these two classes of objects have different luminosity distributions, for example. Stanghellini et al. (2012) compared these two populations of PNe and conclude that Galactic PNe exhibit higher infrared luminosity when compared to LMC-PNe. However, this conclusion may be a selection effect. In fact, their sample of compact Galactic PNe only contains objects with radius smaller than 0.1 parsec, which show $T_{\text {dust }}>10^{2} \mathrm{~K}$. If their radius versus $\log T_{\text {dust }}$ relationship can be extended to larger PNe, objects with $R>0.1$ parsec (and $T_{\text {dust }}<10^{2} \mathrm{~K}$ ) would be added to their sample, and those show $\log \left(L_{\mathrm{IR}} / L_{\odot}\right)<2$. In this case the interval of infrared luminosities of LMC and Galactic PNe would be similar.

A comparison among different distance scales is shown in Fig. 4, where only methods based on the specific intensity of the radiation, i.e. infrared intensity or brightness temperature, have been included. The comparison with the method proposed by SSV (panel a) is interesting because SSV's method was also calibrated using PNe in the LMC. Their model is based on the Shklovsky/Daub distance technique, but with several improvements designed to fix its main limitation: the assumption that 
all PNe are optically thick and thus that they would all have the same ionized mass. According to SSV, the uncertainty of their method is $30 \%$ at least, and this figure cannot be reduced significantly by improving the sample of calibrators, for instance. The uncertainty of the present method is $d_{-37 \%}^{+58 \%}$, but this figure may be greater if the apparent size is misevaluated, for example. A comparison between the results produced by these two methods must consider that there are important differences between them: SSV's is based on the $5 \mathrm{GHz}$ brightness temperature (or its equivalent $\mathrm{H} \beta$ flux) and the nebular size is defined by the photometric radius, i.e. the isocontour that contains $85 \%$ of the nebular flux. Figure 4a shows that these two methods are in good agreement within their respective uncertainties. The case of H1-7 might have been caused by its misidentification by SSV.

The comparison with the method proposed by Phillips (2004) is shown in Fig. 4b. The uncertainty of that method is estimated as $35 \%$, on average. The plot reveals the existence of a bias over the whole range of distances, which makes that distance scale "short" when compared with the present method. However, a similar bias appears when Phillips' method is compared with SSV (Fig. 4c). This suggests that this bias is probably restricted to Phillips' method. The present distance scale agrees more with the distance scale proposed by van de Steene \& Zijlstra (1994, Fig. 4d), which was calibrated with Galactic bulge PNe, assuming that their average distance is $8 \mathrm{kpc}$. The uncertainty of that method is estimated by the authors as $40 \%$, on average. A closer inspection of the original data reveals the cause of the larger differences between the two methods: Pe2-12 $\left(d=8.51 \mathrm{kpc}, d_{\mathrm{vdSZ}}=13.59 \mathrm{kpc}\right), \operatorname{Pe} 1-15(d=6.09 \mathrm{kpc}$, $\left.d_{\mathrm{vdSZ}}=8.47 \mathrm{kpc}\right)$, and Pe1-6 $\left(d=2.43 \mathrm{kpc}, d_{\mathrm{vdSZ}}=4.27 \mathrm{kpc}\right)$. The difference in the distance of Pe2-12 is probably due to an error in the measurement of the flux density at $5 \mathrm{GHz}, 2 \mathrm{mJy}$, the lowest value among all $\mathrm{PNe}$ in the bulge. The difference observed in the case of Pe1-15 may have been caused by incorrect determination of its apparent size: observations at $5 \mathrm{GHz}$ point out a diameter of only $4.8^{\prime \prime}$, whereas Spitzer observations at $8 \mu \mathrm{m}$ suggest $11.3^{\prime \prime}$. The distance produced by both methods would show better agreement if similar apparent diameters were considered. The discrepancy between the sizes at $8 \mu \mathrm{m}\left(48.6^{\prime \prime}\right)$ and at $5 \mathrm{GHz}\left(7.2^{\prime \prime}\right)$ seems to be also the problem with the distance of Pe1-6. These two latter examples illustrate the sensitivity of the method well with respect to the determination of $\theta_{8} \mu \mathrm{m}$.

\section{Conclusions}

A new distance scale was proposed that is based on a relationship between the specific intensity of the nebular radiation at $8 \mu \mathrm{m}$ and its diameter. The calibration, based on a sample of $56 \mathrm{PNe}$ in the LMC, shows a one-sigma spread of 0.2 dex in the nebular size.

Two main sources of error in the method are (1) the intrinsic spread of 0.2 dex in the $D$ versus $I_{8} \mu \mathrm{m}$ relationship; and (2) the misevaluation of the angular diameter. The former causes the distance scale to be accurate to $d_{-37 \%}^{+58 \%}$. An uncertainty of $20 \%$ in the apparent diameter alone generates an additional error of $d_{-10 \%}^{+13 \%}$.

Distances of PNe in the LMC show one-sigma uncertainty of $20 \%$. A comparison between the new method and the distance scale proposed by Stanghellini et al. (2008) does not show significant bias, and they generally agree with each other within $30 \%$.
Acknowledgements. This research made use of the SIMBAD database, operated at the CDS, Strasbourg, France, and the NASA Astrophysics Data System. This work is partially based on observations made with the Spitzer Space Telescope, which is operated by the Jet Propulsion Laboratory, California Institute of Technology, under a contract with NASA. The author acknowledges the grant awarded by FAPESP (proc. 2010/18835-3) entitled "Planetary nebulae, stars, and the chemical evolution of stellar systems". The author thanks the anonymous referee for the excellent critical review of the original manuscript.

\section{References}

Bedding, T. R., \& Zijlstra, A. A. 1994, A\&A, 283, 955

Benedict, G. F., MacArthur, B. E., Napiwotski, R., et al. 2009, AJ, 138, 1969

Cerrigone, L., Hora, J. L., Umana, G., \& Trigilio, C. 2008, ApJ, 682, 1047

Cohen, M., Parker, Q. A., Green, A. J., et al. 2011, MNRAS, 413, 514

Cox A. N. 2000, Allen's Astrophysical Quantities (Springer)

Gutiérrez-Moreno, A., Anguita, C., Loyola, P., \& Moreno, H. 1999, PASP, 111, 1163

Guzmán-Ramírez L., Gómez, Y., Loinard, L., \& Tafoya, D. 2011, MNRAS, 414 3129

Harris, H. C., Dahn, C. C., Canzian, B., et al. 2007, AJ, 133, 631

Heap, S. R., Boggess, A., Holm, A., et al. 1978, Nature, 275, 385

Hora, J. L., Cohen, M., Ellis, R. G., et al. 2008, AJ, 135, 726

Jewitt, D. C., Danielson, G. E., \& Kupferman, P. N. 1986, ApJ, 302, 727

Kwok, S. 1982, ApJ, 258, 280

Kwok, S., Purton, C. R., \& FitzGerald, P. M. 1978, ApJ, 219, L125

Kwok, S., Zhang, Y., Koning, N., Huang, H. H., \& Churchwell, E. 2008, ApJS, 174,426

Li, A., \& Greenberg, J. M. 1997, A\&A, 323, 566

Meade, M. R., Whitney, B. A., Babler, B. L., et al. 2009, Glimpse II - v. 2.0 data release, 31

Meixner, M., Gordon, K. D., Indebetouw, R., et al. 2006, AJ, 132, 2268

Mellema, G. 2004, A\&A, 416, 623

Méndez, R. H., Kudritzki, R. P., Herrero, A., Husfeld, D., \& Groth, H. G. 1988, A\&A, 190, 113

Milne, D. K., \& Aller, L. H. 1975, A\&A, 38, 183

Mould, J. R., Huchra, J. P., Freedman, W. L., et al. 2000, ApJ, 529, 786

Mufson, S. L., Lyon, J., \& Marionni, P. A. 1975, ApJ, 201, L85

Ortiz, R., Copetti, M. V. F., \& Lorenz-Martins, S. 2011, MNRAS, 418, 2004

Phillips, J. P. 2002, ApJS, 138, 199

Phillips, J. P. 2004, MNRAS, 353, 589

Phillips, J. P., \& Ramos-Larios, G. 2008a, MNRAS, 383, 1029

Phillips, J. P., \& Ramos-Larios, G. 2008b, MNRAS, 386, 995

Phillips, J. P., \& Ramos-Larios, G. 2010, MNRAS, 405, 2179

Phillips, J. P., Ramos-Larios, G., Schoereder, K.-P., \& Verbena Contreras, J. L. 2009, MNRAS, 399, 1126

Quino-Mendoza, J. A., Phillips, J. P., \& Ramos-Lario, G. 2011, Rev. Mex. Astron. Astrofis., 47, 31

Ramos-Lario, G., \& Phillips, J. P. 2008, MNRAS, 390, 1014

Ramos-Lario, G., \& Phillips, J. P. 2009, MNRAS, 400, 575

Ramos-Lario, G., \& Phillips, J. P. 2012, MNRAS, 425, 1091

Ramos-Lario, G., Guerrero, M. A., Vazquez, R., \& Phillips, J. P. 2012, MNRAS, 420, 1977

Ruffle, P. M. E., Zijlstra, A. A., Walsh, J. R., et al. 2004, MNRAS, 353, 796

Schoenberner, D., Jacob, R., \& Steffen, M. 2005, A\&A, 441, 573

Schultheis, M., Ganesh, S., Simon, G., et al. 1999, A\&A, 349, L69

Shaw, R. A., Stanghellini, L., Mutchler, M., Balick, B., \& Blades, J. C. 2001, ApJ, 548, 727

Shaw, R. A., Stanghellini, L., Villaver, E., \& Mutchler, M. 2006, ApJS, 167, 201

Shaw, R. A., Reid, W. A., \& Parker, Q. A. 2007, PASP, 119, 19

Shklovsky, I. 1956, Astron. Zh., 33, 222

Siódmiak, N., \& Tylenda, R. 2001, A\& A, 373, 1032

Stanghellini, L., Shaw, R. A., \& Villaver, E. 2008, ApJ, 689, 194 (SSV)

Stanghellini, L., García-Hernández, D. A., García-Lario, P., et al. 2012, ApJ, 753,172

Tylenda, R., Siódmiak, N., Górny, S. K., Corradi, R. L. M., \& Schwarz, H. E. 2003, A\&A, 405, 627

Urosevic, D., Vukotic, B., Arbutina, B., et al. 2009, A\&A, 495, 537

van de Steene, G. C., \& Zijlstra, A. A. 1994, A\&AS, 108, 485

van de Steene, G. C., \& Zijlstra, A. A. 1995, A\&A, 293, 541

Volk, K. 1992, ApJS, 80, 347

Wareing, C. J., Zijlstra, A. A., \& O’Brien, T. J. 2007, MNRAS, 382, 1233

Zhang, C. Y., \& Kwok, S. 1993, ApJS, 88, 137

Pages 7 to 10 are available in the electronic edition of the journal at http://www . aanda. org 
R. Ortiz: A revised distance scale of planetary nebulae $(R N)$

Table 1. Flux density and apparent diameters of Galactic PNe observed during the Spitzer survey.

\begin{tabular}{lcccccll}
\hline \hline PNG name & $l(\mathrm{deg})$ & $b(\mathrm{deg})$ & $F_{8 \mu \mathrm{m}}(\mathrm{Jy})$ & $\theta_{8 \mu \mathrm{m}}\left({ }^{\prime \prime}\right)$ & $\left.\theta_{\text {vis }}{ }^{\prime \prime}\right)$ & Other names & References \\
\hline PNG002.4+05.8 & 002.432 & +05.847 & - & 41.5 & 32.8 & NGC 6369 & RLA12, Ty103 \\
PNG003.5-02.4 & 003.553 & -02.442 & 0.3557 & 18.0 & 18.3 & IC 4673 & QPR11, Ty103 \\
PNG008.0+03.9 & 008.076 & +03.905 & 0.5690 & 36.5 & 40.5 & NGC 6445 & PRL10, Ty103 \\
PNG009.4-05.0 & 009.407 & -05.049 & - & 21.0 & 16.0 & NGC 6629 & RLP09, Ty103 \\
PNG010.8-01.8 & 010.818 & -01.827 & 0.6068 & 16.3 & 11.9 & NGC 6578 & QPR11, Ty103 \\
PNG011.7-00.6 & 011.744 & -00.650 & 1.3902 & 11.0 & 7.2 & NGC 6567 & PRL08a, Ty103 \\
PNG027.7+00.7 & 027.702 & +00.705 & 0.1442 & 9.8 & 8.5 & M 2-45 & PRL08a, Tyl03 \\
PNG033.1-06.3 & 033.159 & -06.388 & - & 110.0 & 75.6 & NGC 6772 & RLP09, Ty103 \\
PNG040.3-00.4 & 040.370 & -00.475 & 0.0927 & 35.6 & 31.5 & A 53 & PRL08a \\
PNG061.4-09.5 & 061.491 & -09.571 & 0.1510 & 48.7 & 39.3 & NGC 6905 & PRL10 \\
PNG189.1+19.8 & 189.156 & +19.843 & - & 52.1 & 45.4 & NGC 2371 & RLP12, RLP12 \\
PNG231.8+04.1 & 231.802 & +04.117 & - & 90.0 & 79.5 & NGC 2438 & RLP09, Ty103 \\
PNG234.8+02.4 & 234.836 & +02.420 & - & 21.0 & 38.4 & NGC 2440 & RLP09, Ty103 \\
PNG261.0+32.0 & 261.051 & +32.050 & - & 67.3 & 31.1 & NGC 3242 & Phi09 \\
PNG300.5-01.1 & 300.589 & -01.109 & 0.0653 & 10.3 & 8.5 & Hen 2-85 & PRL08a, Ty103 \\
PNG306.4-00.6 & 306.416 & -00.688 & 0.1955 & 23.0 & 26.0 & Th 2-A & PRL08a, Tyl03 \\
PNG315.0-00.3 & 315.031 & -00.370 & 0.1833 & 26.7 & 20.6 & Hen 2-111 & PRL08a \\
PNG336.2+01.9 & 336.220 & +01.973 & 0.1104 & 48.7 & 9.4 & Pe 1-6 & QPR11, Ty103 \\
PNG345.2-01.2 & 345.277 & -01.249 & 4.8204 & 58.8 & 9.6 & H 1-7 & QPR11 \\
PNG349.5+01.0 & 349.508 & +01.056 & 17.3412 & 98.4 & 55.9 & NGC 6302 & PRL08a \\
PNG356.5-02.3 & 356.531 & -02.394 & 1.1248 & 9.2 & 6.5 & M 1-27 & QPR11, Ty103 \\
\hline
\end{tabular}

References. Tyl03 = Tylenda et al. (2003); PRL08a = Phillips \& Ramos-Larios (2008); RLP09 = Ramos-Lario \& Phillips (2009); Phi09 = Phillips et al. (2009); PRL10 = Phillips \& Ramos-Lario (2010); QPR11 = Quino-Mendoza et al. (2011); RLA12 = Ramos-Lario et al. (2012); RLP12 = Ramos-Lario \& Phillips (2012). 
Table 2. PNe in the LMC and their characteristics.

\begin{tabular}{|c|c|c|c|c|c|c|c|}
\hline $\mathrm{RA}(\mathrm{J} 2000)$ & $\operatorname{Dec}(\mathrm{J} 2000)$ & $m_{8 \mu \mathrm{m}}$ & $\theta_{\text {vis }}\left({ }^{\prime \prime}\right)$ & $1.23 \times \theta_{\text {vis }}\left({ }^{\prime \prime}\right)$ & $\theta_{8 \mu \mathrm{m}}\left({ }^{\prime \prime}\right)$ & type & LMC name \\
\hline 44808.7 & -672606.3 & $10.32 \pm 0.01$ & 0.480 & 0.590 & 0.660 & 1 & SMP5 \\
\hline 45024.7 & -681316.0 & $11.09 \pm 0.01$ & 0.820 & 1.009 & 0.755 & 1 & SMP9 \\
\hline 45109.0 & -684905.4 & $13.69 \pm 0.07$ & 1.580 & 1.943 & 1.967 & 3 & SMP10 \\
\hline 45137.9 & -670516.3 & $5.92 \pm 0.00$ & 0.646 & 0.795 & 0.812 & 2 & SMP11 \\
\hline 50000.2 & -702740.5 & $9.76 \pm 0.01$ & 0.810 & 0.996 & 1.298 & 0 & SMP13 \\
\hline 50202.0 & -694853.6 & $12.41 \pm 0.03$ & 1.340 & 1.648 & 1.697 & 2 & SMP16 \\
\hline 50342.7 & -700646.8 & $12.38 \pm 0.04$ & 0.660 & 0.812 & 0.741 & 0 & SMP18 \\
\hline 50341.3 & -701353.0 & $10.29 \pm 0.01$ & 0.720 & 0.886 & 0.909 & 1 & SMP19 \\
\hline 50623.9 & -690319.3 & $9.02 \pm 0.01$ & 0.405 & 0.498 & 0.483 & 0 & SMP25 \\
\hline 50754.9 & -665745.3 & $12.42 \pm 0.03$ & 0.760 & 0.935 & 0.679 & 3 & SMP27 \\
\hline 50757.7 & -685146.2 & $10.39 \pm 0.01$ & 0.450 & 0.554 & 0.484 & 3 & SMP28 \\
\hline 50803.5 & -684016.3 & $9.30 \pm 0.01$ & 0.490 & 0.603 & 0.486 & 2 & SMP29 \\
\hline 50910.6 & -665338.2 & $13.24 \pm 0.05$ & 1.466 & 1.803 & 1.528 & 2 & SMP30 \\
\hline 50920.2 & -674724.3 & $7.63 \pm 0.00$ & 0.260 & 0.320 & 0.220 & 0 & SMP31 \\
\hline 51017.2 & -684822.5 & $11.05 \pm 0.02$ & 0.534 & 0.657 & 0.866 & 1 & SMP34 \\
\hline 51102.9 & -674758.8 & $9.49 \pm 0.01$ & 0.464 & 0.571 & 0.715 & 1 & SMP37 \\
\hline 51142.1 & -683459.7 & $9.89 \pm 0.01$ & 0.575 & 0.707 & 0.615 & 1 & SMP39 \\
\hline 51920.7 & -665807.5 & $12.16 \pm 0.03$ & 1.640 & 2.017 & 1.918 & 2 & SMP45 \\
\hline 51929.7 & -685107.9 & $10.95 \pm 0.01$ & 0.538 & 0.662 & 0.805 & 1 & SMP46 \\
\hline 51954.7 & -693104.5 & $8.81 \pm 0.01$ & 0.379 & 0.466 & 0.437 & 1 & SMP47 \\
\hline 52009.7 & -695338.9 & $8.84 \pm 0.01$ & 0.374 & 0.460 & 0.638 & 1 & SMP48 \\
\hline 52009.4 & -702538.1 & $13.76 \pm 0.07$ & 1.000 & 1.230 & 1.250 & 0 & SMP49 \\
\hline 52123.8 & -683534.4 & $10.93 \pm 0.01$ & 0.730 & 0.898 & 1.024 & 0 & SMP52 \\
\hline 52132.9 & -670004.0 & $11.80 \pm 0.02$ & 0.504 & 0.620 & 0.648 & 1 & SMP53 \\
\hline 52420.9 & -700500.5 & $8.05 \pm 0.00$ & 0.230 & 0.283 & 0.288 & 0 & SMP58 \\
\hline 52427.4 & -702223.7 & $12.71 \pm 0.03$ & 3.137 & 3.859 & 3.649 & 3 & SMP59 \\
\hline 52455.0 & -713255.4 & $9.83 \pm 0.01$ & 0.492 & 0.605 & 0.671 & 1 & SMP62 \\
\hline 52526.2 & -685553.8 & $10.28 \pm 0.01$ & 0.599 & 0.737 & 0.881 & 1 & SMP63 \\
\hline 52743.8 & -712556.0 & $11.55 \pm 0.02$ & 0.590 & 0.726 & 0.826 & 0 & SMP65 \\
\hline 52915.7 & -673246.7 & $11.04 \pm 0.01$ & 0.733 & 0.902 & 0.948 & 2 & SMP67 \\
\hline 52902.9 & -701924.8 & $12.67 \pm 0.03$ & 1.136 & 1.397 & 1.000 & 1 & SMP68 \\
\hline 52923.2 & -671321.9 & $13.19 \pm 0.06$ & 1.622 & 1.995 & 2.325 & 2 & SMP69 \\
\hline 53033.3 & -704437.6 & $8.84 \pm 0.01$ & 0.522 & 0.642 & 0.550 & 1 & SMP71 \\
\hline 53122.0 & -704044.9 & $8.42 \pm 0.00$ & 0.289 & 0.356 & 0.324 & 1 & SMP73 \\
\hline 53329.8 & -715228.4 & $9.10 \pm 0.01$ & 0.705 & 0.867 & 0.668 & 1 & SMP74 \\
\hline 53347.0 & -683644.2 & $7.46 \pm 0.00$ & 0.330 & 0.406 & 0.348 & 0 & SMP75 \\
\hline 53421.3 & -685824.9 & $8.36 \pm 0.00$ & 0.476 & 0.586 & 0.368 & 1 & SMP78 \\
\hline 53439.0 & -701955.5 & $13.86 \pm 0.08$ & 0.480 & 0.590 & 0.662 & 0 & SMP80 \\
\hline 53557.6 & -695816.6 & $11.40 \pm 0.02$ & 0.305 & 0.375 & 0.341 & 1 & SMP82 \\
\hline 53620.8 & -671807.5 & $11.80 \pm 0.02$ & 3.801 & 4.675 & 4.763 & 2 & SMP83 \\
\hline 53653.0 & -715338.0 & $11.06 \pm 0.01$ & 0.523 & 0.643 & 0.380 & 1 & SMP84 \\
\hline 54233.3 & -702924.0 & $11.46 \pm 0.02$ & 0.524 & 0.645 & 0.649 & 1 & SMP88 \\
\hline 54237.0 & -700931.1 & $8.80 \pm 0.01$ & 0.479 & 0.589 & 0.378 & 1 & SMP89 \\
\hline 54506.0 & -680650.9 & $13.18 \pm 0.05$ & 1.627 & 2.001 & 2.324 & 2 & SMP91 \\
\hline 54704.7 & -692733.3 & $9.31 \pm 0.01$ & 0.579 & 0.712 & 0.662 & 1 & SMP92 \\
\hline 54938.8 & -690959.3 & $10.90 \pm 0.02$ & 3.286 & 4.042 & 3.295 & 2 & SMP93 \\
\hline 60145.3 & -675606.4 & $11.66 \pm 0.02$ & 1.045 & 1.285 & 1.341 & 1 & SMP95 \\
\hline 50643.7 & -691537.8 & $13.02 \pm 0.05$ & 1.660 & 2.042 & 2.298 & 2 & Sa107 \\
\hline 51148.1 & -692343.1 & $9.37 \pm 0.01$ & 1.198 & 1.474 & 1.696 & 1 & Sa109 \\
\hline 52456.7 & -691531.2 & $11.66 \pm 0.04$ & 1.239 & 1.524 & 1.592 & 2 & Sa117 \\
\hline 52117.6 & -694301.0 & $12.66 \pm 0.07$ & 1.655 & 2.036 & 1.690 & 1 & Sa115 \\
\hline 50427.7 & -685811.4 & $11.65 \pm 0.03$ & 1.580 & 1.943 & 2.168 & 0 & MG14 \\
\hline 52235.3 & -682425.3 & $12.17 \pm 0.03$ & 0.354 & 0.435 & 0.497 & 1 & MG40 \\
\hline 52834.4 & -703301.6 & $12.62 \pm 0.03$ & 1.321 & 1.625 & 1.318 & 1 & MG51 \\
\hline 53330.9 & -690813.3 & $12.82 \pm 0.06$ & 1.175 & 1.445 & 1.677 & 0 & MG60 \\
\hline 53853.6 & -695755.7 & $12.71 \pm 0.10$ & 1.052 & 1.294 & 0.984 & 1 & Mo36 \\
\hline
\end{tabular}

Notes. Spitzer $8 \mu \mathrm{m}$ magnitudes were extracted from Hora et al. (2008) and visual diameters $\left(\theta_{\text {vis }}\right)$ from Shaw et al. (2001, 2006). Type (7th column) refers to the following classification: $0=$ round; $1=$ elliptical; $2=$ bipolar; $3=$ other. 
R. Ortiz: A revised distance scale of planetary nebulae $(R N)$

Table 3. Spitzer $8 \mu \mathrm{m}$ magnitudes, diameters and distances of Galactic PNe determined with the present method (d), Stanghellini et al. 2008 (SSV, $\left.d_{\mathrm{SSV}}\right)$, Phillips $2004\left(d_{\mathrm{Phil}}\right)$, and van de Steene \& Zijlstra $1994\left(d_{\mathrm{vdSZ}}\right)$.

\begin{tabular}{|c|c|c|c|c|c|c|c|c|c|c|}
\hline PNG name & $\begin{array}{c}l \\
(\mathrm{deg})\end{array}$ & $\begin{array}{c}b \\
(\mathrm{deg}) \\
\end{array}$ & $m_{8 \mu \mathrm{m}}$ & $\begin{array}{c}\theta_{8 \mu \mathrm{m}} \\
\left({ }^{\prime \prime}\right)\end{array}$ & $\begin{array}{c}d \\
(\mathrm{kpc})\end{array}$ & $\begin{array}{l}d_{\mathrm{SSV}} \\
(\mathrm{kpc})\end{array}$ & $\begin{array}{r}d_{\text {Phil }} \\
(\mathrm{kpc})\end{array}$ & $\begin{array}{l}d_{\mathrm{vdSZ}} \\
(\mathrm{kpc})\end{array}$ & Other names & $\begin{array}{l}\text { References and } \\
\text { comments }\end{array}$ \\
\hline $002.8-02.2$ & 002.856 & -02.292 & 9.71 & 14.3 & 8.51 & 12.19 & - & 13.59 & Pe 2-12 & $\begin{array}{l}\text { QPR11 } \\
\theta_{8} \text { from text, Ruf04 }\end{array}$ \\
\hline $003.5-02.4$ & 003.553 & -02.442 & 5.64 & 17.6 & 3.22 & 3.22 & - & 2.83 & IC 4673 & QPR11, Tyl03 \\
\hline $003.9-02.3$ & 003.923 & -02.323 & 5.17 & 7.2 & 4.74 & 6.09 & - & 4.46 & M 1-35 & QPR11 \\
\hline $008.0+03.9$ & 008.076 & +03.905 & 5.13 & 36.3 & 1.95 & 1.38 & - & 1.24 & NGC 6445 & $\begin{array}{l}\text { PRL10, Tyl03 } \\
\text { bipolar PN }\end{array}$ \\
\hline $009.8-01.1$ & 009.808 & -01.145 & 7.75 & 8.3 & 7.57 & - & - & - & PHR1811-2100 & QPR11 \\
\hline $010.1+00.7$ & 010.099 & +00.739 & 3.16 & 60.3 & 0.98 & - & 1.77 & 1.60 & NGC 6537 & PRL08a \\
\hline $010.2+00.4$ & 010.211 & +00.429 & 3.29 & 23.7 & 1.67 & - & - & - & IRAS18036-1954 & PRL08b \\
\hline $010.3+00.5$ & 010.393 & +00.538 & 3.86 & 19.7 & 2.08 & - & - & - & IRAS18036-1941 & $\begin{array}{l}\text { PRL08b } \\
\text { irregular PN }\end{array}$ \\
\hline $010.8-01.8$ & 010.818 & -01.827 & 5.06 & 15.9 & 3.01 & 3.64 & 2.31 & 2.36 & NGC 6578 & QPR11, Tyl03 \\
\hline $011.7-00.6$ & 011.744 & -00.650 & 4.16 & 10.4 & 3.13 & 3.61 & 2.40 & 2.43 & NGC 6567 & PRL08a, Tyl03 \\
\hline $018.2-00.9$ & 018.241 & -00.915 & 7.69 & 14.9 & 5.43 & - & - & - & MPA 1827-132 & $\begin{array}{l}\text { RLP08 } \\
\theta_{8} \mu \mathrm{m} \text { from text }\end{array}$ \\
\hline $022.5+01.0$ & 022.570 & +01.055 & 7.62 & 16.5 & 5.06 & - & - & - & Mac 1-13 & PRL08a \\
\hline $025.9-02.1$ & 025.911 & -02.184 & 7.51 & 11.3 & 6.09 & 8.25 & 7.40 & 8.47 & Pe $1-15$ & QPR11 \\
\hline $027.7+00.7$ & 027.702 & +00.705 & 6.62 & 9.1 & 5.65 & 4.50 & 2.64 & 2.63 & M 2-45 & PRL08a, Tyl03 \\
\hline $029.0+00.4$ & 029.078 & +00.454 & 6.46 & 71.9 & 1.79 & - & - & - & A 48 & $\begin{array}{l}\text { PRL08a } \\
\text { outer limits uncertain }\end{array}$ \\
\hline $029.2-00.0$ & 029.211 & -00.069 & 5.83 & 8.2 & 5.08 & - & - & - & TDC 1 & PRL08a \\
\hline $031.6+00.3$ & 031.662 & +00.366 & 4.48 & 8.7 & 3.69 & - & - & - & IRAS18452-0191 & $\begin{array}{l}\text { PRL08b } \\
\theta_{8 \mu \mathrm{m}} \text { from isocontours }\end{array}$ \\
\hline $032.9+00.4$ & 032.990 & +00.040 & 3.36 & 36.8 & 1.33 & - & - & - & IRAS $18488+0000$ & $\begin{array}{l}\text { PRL08b } \\
\theta_{8 \mu \mathrm{m}} \text { from isocontours }\end{array}$ \\
\hline $040.3-00.4$ & 040.370 & -00.475 & 7.10 & 35.4 & 3.00 & 1.92 & 1.68 & - & A 53 & PRL08a \\
\hline $049.6+00.8$ & 049.695 & +00.864 & 4.32 & 5.7 & 4.50 & - & - & - & IRAS19172+1511 & PRL08b \\
\hline 061.4-09.5 & 061.491 & -09.571 & 6.57 & 48.6 & 2.26 & 1.75 & 1.62 & 2.22 & NGC 6905 & $\begin{array}{l}\text { PRL10 } \\
\text { PN with knots }\end{array}$ \\
\hline $062.4-00.2$ & 062.494 & -00.270 & 8.45 & 6.6 & 9.91 & 10.16 & 7.00 & - & M 2-48 & $\begin{array}{l}\text { PRL08a } \\
\theta_{8 \mu \mathrm{m}} \text { uncertain }\end{array}$ \\
\hline $215.6+03.6$ & 215.699 & +03.621 & 2.96 & 26.8 & 1.46 & 1.37 & - & 1.78 & NGC 2346 & PRL10 \\
\hline $261.9+08.5$ & 261.983 & +08.584 & 7.01 & 37.8 & 2.84 & 2.00 & 1.79 & 2.56 & NGC 2818 & $\begin{array}{l}\text { PRL10 } \\
\text { irregular PN, Tyl03 }\end{array}$ \\
\hline $292.6+01.2$ & 292.656 & +01.238 & 7.72 & 62.4 & 2.52 & 1.62 & 1.54 & 2.05 & NGC 3699 & $\begin{array}{l}\text { PRL10 } \\
\text { irregular PN, Ty103 }\end{array}$ \\
\hline $296.8-00.9$ & 296.849 & -00.985 & 8.48 & 16.1 & 6.15 & - & - & - & PHR J1157-6312 & Kwo08, RLP08 \\
\hline $297.0+01.1$ & 297.050 & +01.096 & 9.45 & 18.7 & 6.97 & - & - & - & PHR 1202-611 & $\begin{array}{l}\text { Coh11, RLP08 } \\
\theta_{8 \mu \mathrm{m}} \text { from isocontours }\end{array}$ \\
\hline $297.0+01.1$ & 297.050 & +01.096 & 9.45 & 16.1 & 7.55 & - & - & - & PHR 1202-611 & Coh11, RLP08 \\
\hline $299.2+01.0$ & 299.216 & +01.075 & 9.67 & 12.5 & 9.07 & - & - & - & PHR 1220-613 & $\begin{array}{l}\text { Coh11, RLP08 } \\
\theta_{8 \mu \mathrm{m}} \text { from isocontours }\end{array}$ \\
\hline $300.5-01.1$ & 300.589 & -01.109 & 7.48 & 9.7 & 6.57 & 3.56 & 2.53 & - & Hen 2-85 & PRL08a, Tyl03 \\
\hline $302.3-00.5$ & 302.374 & -00.541 & 6.99 & 17.6 & 4.27 & - & - & - & PHR 1246-6324 & Kwo08, RLP08 \\
\hline $306.4-00.6$ & 306.416 & -00.688 & 6.29 & 22.7 & 3.22 & 2.47 & 2.07 & 2.57 & Th 2-A & PRL08a, Tyl03 \\
\hline $315.0-00.3$ & 315.031 & -00.370 & 6.36 & 26.5 & 3.01 & 3.51 & 2.66 & - & Hen 2-111 & PRL08a \\
\hline $319.6+15.7$ & 319.687 & +15.740 & 6.80 & 89.9 & 1.70 & 2.38 & 1.50 & 1.87 & IC 4406 & C08, Ty103 \\
\hline $328.3+00.7$ & 328.357 & 00.766 & 7.02 & 26.8 & 3.43 & - & - & - & PHR1552-5254 & $\begin{array}{l}\text { Coh11, RLP08 } \\
\theta_{8} \mu \mathrm{m} \text { uncertain }\end{array}$ \\
\hline $333.9+00.6$ & 333.930 & +00.686 & 4.56 & 13.5 & 2.96 & - & - & - & PHR J1619-4913 & $\begin{array}{l}\text { Kwo08, RLP08 } \\
\text { inner shell size }\end{array}$ \\
\hline $336.2+01.9$ & 336.220 & +01.973 & 6.91 & 48.6 & 2.43 & 5.38 & - & 4.27 & Pe 1-6 & QPR11, Ty103 \\
\hline $342.7+00.7$ & 342.744 & +00.752 & 8.70 & 8.7 & 9.00 & - & - & - & Н 1-3, На 2-3 & $\begin{array}{l}\text { PRL08a } \\
\theta_{8 \mu \mathrm{m}} \text { from isocontours }\end{array}$ \\
\hline
\end{tabular}

Notes. Nebular diameters were taken from $8 \mu \mathrm{m}$ intensity profiles, except when noted.

References. Tyl03 = Tylenda et al. (2003); Ruf04 = Ruffle et al. (2004); C08 = Cerrigone et al. (2008); PRL08a = Phillips \& Ramos-Larios (2008a); PRL08b = Phillips \& Ramos-Larios (2008b); RLP08 = Ramos-Lario \& Phillips (2008); Kwo08 = Kwok et al. (2008); PRL10 = Phillips $\&$ Ramos-Larios (2010); Coh11 = Cohen et al. (2011); QPR11 = Quino-Mendoza et al. (2011). 
A\&A 560, A85 (2013)

Table 3. continued.

\begin{tabular}{|c|c|c|c|c|c|c|c|c|c|c|}
\hline PNG name & $\begin{array}{c}l \\
(\mathrm{deg})\end{array}$ & $\begin{array}{c}b \\
(\mathrm{deg})\end{array}$ & $m_{8 \mu \mathrm{m}}$ & $\begin{array}{c}\theta_{8 \mu \mathrm{m}} \\
\left({ }^{\prime \prime}\right)\end{array}$ & $\begin{array}{c}d \\
(\mathrm{kpc})\end{array}$ & $\begin{array}{l}d_{\mathrm{SSV}} \\
(\mathrm{kpc})\end{array}$ & $\begin{array}{l}d_{\text {Phil }} \\
(\mathrm{kpc})\end{array}$ & $\begin{array}{l}d_{\mathrm{vdSZ}} \\
(\mathrm{kpc})\end{array}$ & Other names & $\begin{array}{l}\text { References and } \\
\text { comments }\end{array}$ \\
\hline $343.9+00.8$ & 343.992 & +00.835 & 5.04 & 8.5 & 4.21 & - & - & - & Н 1-5, На 2-5 & PRL08a \\
\hline $344.2-01.2$ & 344.246 & -01.214 & 6.01 & 25.2 & 2.87 & - & - & - & H 1-6, Ha 2-6 & QPR11 \\
\hline $344.4+01.8$ & 344.407 & +01.814 & 9.20 & 29.8 & 5.13 & - & - & - & MPA1654-4041 & $\begin{array}{l}\text { QPR11 } \\
\text { highly eccentric PN }\end{array}$ \\
\hline $345.2-01.2$ & 345.277 & -01.249 & 2.81 & 58.7 & 0.92 & 10.02 & - & - & Н 1-7, Нa 2-7 & QPR11 \\
\hline $346.6+00.8$ & 346.683 & +00.855 & 8.35 & 14.1 & 6.44 & - & - & - & IRAS $17025-3925$ & PRL08b \\
\hline $349.5+01.0$ & 349.508 & +01.056 & 1.42 & 98.3 & 0.52 & 0.74 & 0.52 & 0.54 & NGC 6302 & $\begin{array}{l}\text { PRL08a } \\
\text { PN with "spikes" }\end{array}$ \\
\hline $352.8-00.5$ & 352.874 & -00.515 & 5.40 & 9.0 & 4.40 & - & - & - & MPA 1729-3513 & $\begin{array}{l}\text { ZKw09, RLP08 } \\
\theta_{8 \mu \mathrm{m}} \text { from isocontours }\end{array}$ \\
\hline $356.5-02.3$ & 356.531 & -02.394 & 4.39 & 8.5 & 3.67 & 4.61 & - & 3.53 & M 1-27 & QPR11, Tyl03 \\
\hline $359.1-01.7$ & 359.116 & -01.720 & 4.57 & 13.8 & 2.94 & 4.13 & - & 3.16 & M 1-29 & QPR11 \\
\hline
\end{tabular}

